\title{
ESTIMATION OF PRECISION AND UNCERTAINTY OF A CALIBRATION ARTEFACT FOR CMMS
}

\author{
S. D. ANTUNES \\ Instituto Superior Técnico, Av ${ }^{a}$ Rovisco Pais, 1049-001 Lisboa, PORTUGAL \\ E-mail: santunes@ist.utl.pt \\ M. A. F. VICENTE \\ Universidade de Coimbra, Departamento de Matemática da FCTUC, Apartado 3008 \\ 3001-454 Coimbra, PORTUGAL \\ E-mail: vicente@mat.uc.pt

\begin{abstract}
A new kind of artefact, based on a modification of the hexapod machine's well-known structure, has been introduced by Antunes, S. D. et al in [1], in order to determine the global errors of coordinate measuring machines. Here we are presenting results from validation of the technique: using a self-calibrated method and modeling the reference value for calibration based on laser trilateration.
\end{abstract}

\section{Introduction}

Uncertainty, as defined in the ISO Guide to the Expression of Uncertainty in Measurement (GUM) [4] and in the International Vocabulary of Basic and General Terms in Metrology (VIM), is a parameter, associated with the result of a measurement, which characterizes the dispersion of the values that could reasonably be attributed to the measurand.

Calibration and numerical error correction of coordinate measuring machines (CMMs) require an error behavior mathematical model and methods to assess the errors. Traceability of CMMs must be based on traceability chains and commonly accepted methods for uncertainty evaluation [3].

\section{Calibration Artefacts for CMMs}

Different artefacts (geometrical gauges) can be used to perform the calibration of large CMMs (with one or more axis length bigger than $2 \mathrm{~m}$ ). Between the calibrated artefacts, we can refer the 2D lightweight ball plates (with carbon fiber rod structure and ceramic spheres), the 2D disassemblable ball plates (with L-shape, for example, made of carbon fiber tubes) or the 1D disassemblable multi-ball bars (of carbon fiber pipes), as used by [2]. Alternatively, to perform this task, the calibration can be made with the use of an arrangement of laser 
interferometers and level meters or straight edges to access the full error analysis, but these methods require expensive tools, are time consuming and need especially skilled personnel. In addition, it is possible to use uncalibrated artefacts, but it is necessary to have absolute length standards to determine scale factors. In this last case, it is necessary to place the artefact in substantially more positions then when using calibrated artefacts.

For small and medium size coordinate measuring machines there are several artefact-based methods for full error analysis, which is a prerequisite for establishing traceability. Like the case of large CMMs, there are calibrated and uncalibrated artefacts for this purpose. The most common calibrated artefacts are the gage blocks, step gages, the ball bars, the ball plates and the hole plates ${ }^{\mathrm{a}}$. In addition, laser interferometers are also used in order to perform the measurement of linear accuracy, straightness and angularity checks. Additionally, there are uncalibrated devices, like the artefact presented in [1], which is the object of the main study described in this text.

Artefacts are effective for local and relative calibrations of CMMs. We have compared four different types of reference artefacts [5]:

- the single sphere (see figure 1);

- the step gauge block (see figure 2);

- the ball plate (see figure 3);

- the hole bar (see figure 4).

The single sphere is used to calibrate the probe of the CMM and a good metrological sphere of known and calibrated diameter is needed. All the other listed artefacts are used to calibrate the CMM, in order to find systematic errors on the CMM's measurement volume.

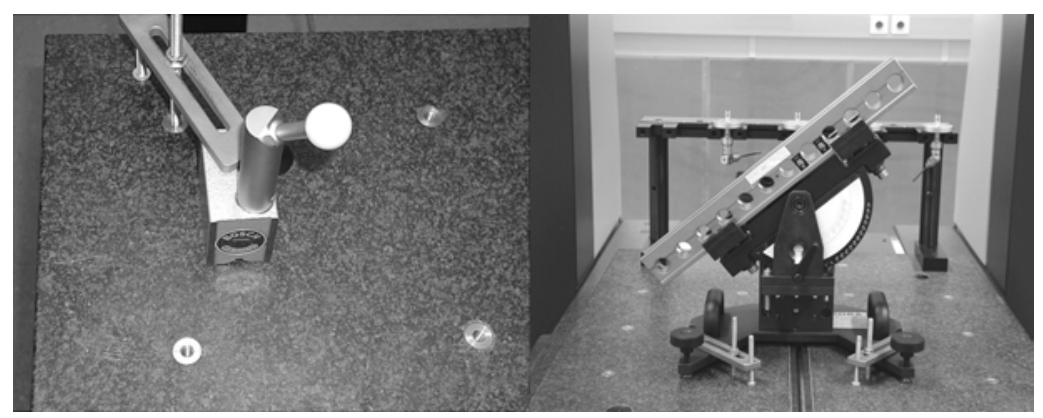

Figure 1. The sphere.

Figure 2. The step gauge block.

${ }^{\text {a }}$ Like the presented artefact in http://www.1gg.com/html/calibcmm.html. 


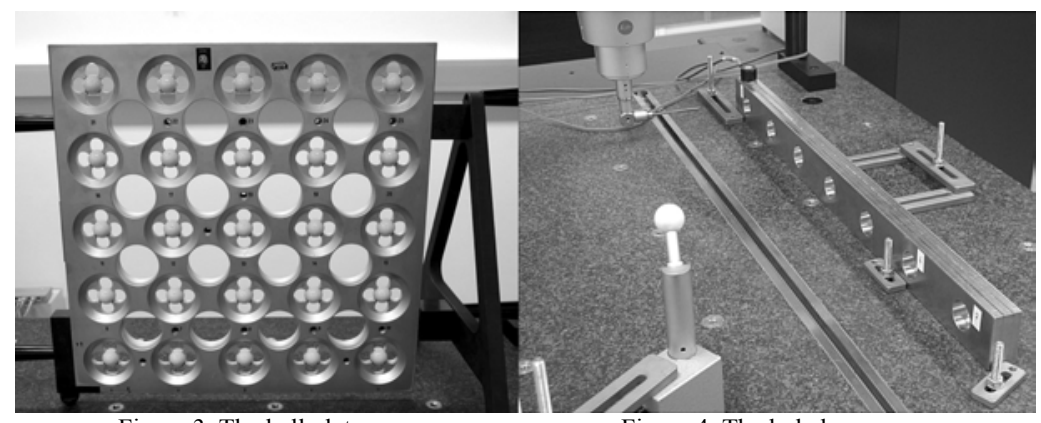

Figure 3. The ball plate.

Figure 4. The hole bar.

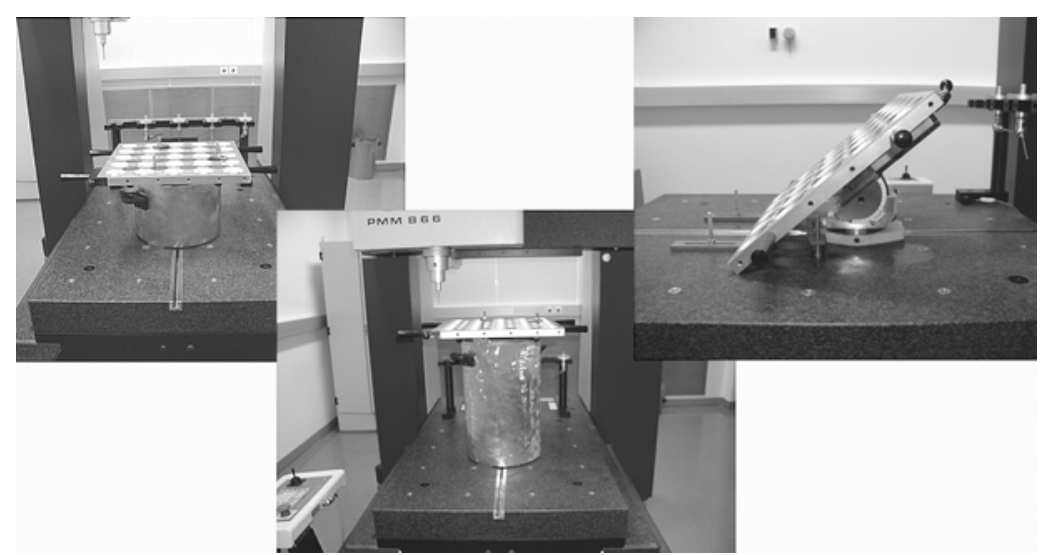

Figure 5. Trying to calibrate CMM's volume measurement with different supports for the ball plate

The step gauge block enables a linear calibration in a direction perpendicular to the reference surface of the gauges.

The ball plate allows the calibration in the plane of the center of the balls constituting the ball plate (the reference points are the center of the balls in its plane).

The hole bar allows a linear calibration in the plane of one surface of the holes (the reference points are the center of the holes in that plane). But the hole bar also enables the measurement of local errors measuring the position of the axis of reference holes in the bar.

We tried to solve the problem of volume calibration with a ball plate, developing specific supports for using the ball plate (see figure 5), but we always had the problem on how correlate measurements with ball plate calibration. 
Those artefacts are effective for local and relative calibrations, but are not appropriate for measuring all calibrating parameters of a CMM. In order to solve those problems, a new kind of artefact, based on a modification of the known structure of the hexapod machine, has been introduced in [1]. The proposed artefact was similar to a tripod system constituted by three links connecting three fixed spheres to a mobile sphere, with the mobile sphere acting as the reference point for CMM calibration (see figure 6, from [1]).

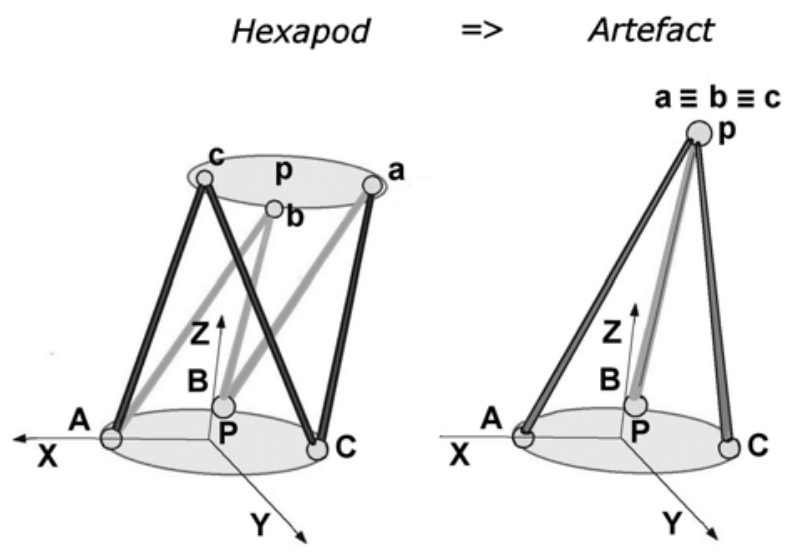

Figure 6. The calibrating artefact, hexapod based.

This kind of artefact needs to be validated, for the purpose of verifying its adequacy. Its validation and the measurement techniques use data fusion applied to non-linear and non-observable systems.

Comparing the calculated position of the moving sphere center with the corresponding measured position by the CMM in the measurement volume does the global calibration of CMMs. Only length variations are used to obtain the calculated position and these length variations are measured by three miniature laser interferometers installed inside the telescopic links (see figures 7 and 8). The artefact uses a self-calibrated method and its modelling is based on laser trilateration.

The problem of global calibration can be divided into two steps:

$1^{\text {st }}$ step - prediction (geometry identification): knowledge about system geometry (localization of fixed points) and tracking (following mobile point) are used to obtain geometry identification (that is done by finding the initial length of the links $l_{i}$, the coordinates of the mobile point $x_{j}, y_{j}$, and the coordinates of the fixed points $x 0_{i}, y 0_{i}$, that minimize: 


$$
\sum_{i=1}^{m} \sum_{j=1}^{n}\left[\left(l_{i}+d l_{i j}\right)-\sqrt{\left(x 0_{i}-x_{j}\right)^{2}+\left(y 0_{i}-y_{j}\right)^{2}}\right]^{2}
$$

with $d l_{i j}{ }^{b}$ representing laser interferometer measurements);

$2^{\text {nd }}$ step - update (system identification): after tracking and identifying geometry, the problem is the identification of the mobile point position, in real time, using 3D laser trilateration.

\section{Modeling Artefact for Global Calibration of CMMs}

Let us consider three fixed spheres $P_{1}, P_{2}$ and $P_{3}$, each one connected to a mobile sphere $Q$ by a telescopic link (see figure 8 ). If we know the lengths of the lines connecting the center of the fixed spheres to the mobile sphere perturbed by known noise and by random noise:

$$
L_{i}=L_{0 i}+p_{c i}+p_{u i}
$$

from trilateration, we can find the coordinates of the mobile sphere $Q(X, Y, Z)$ and also the uncertainty of each coordinate $\left(u_{X}, u_{Y}, u_{Z}\right)$.

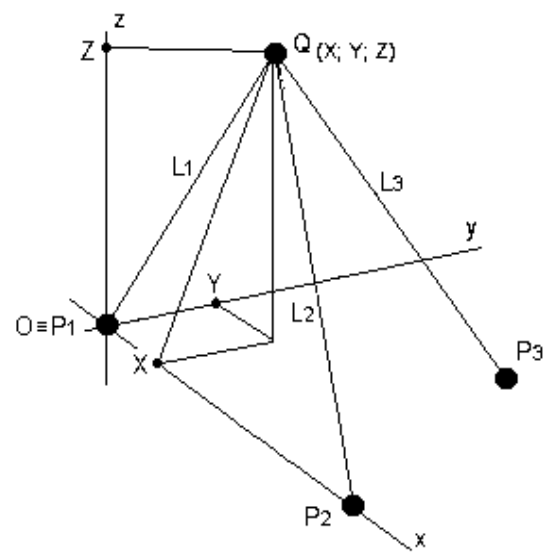

Figure 7. Reference coordinates $O x y z$.

\footnotetext{
${ }^{\mathrm{b}}$ The measurements of links increments, $d l_{i j}$, are perturbed, at least, by ambient humidity, ambient temperature and atmospheric pressure. Also, measured $d l_{i j}$ are estimated with an uncertainty composed by the uncertainties of measured perturbations, which probability density functions are of unknown type gaussian/non-gaussian and, certainly, constituting processes that may be stationary/non-stationary.
} 
Considering the coordinates from the fixed spheres centers $P_{1}\left(X_{1}, Y_{1}, Z_{1}\right)$, $P_{2}\left(X_{2}, Y_{2}, Z_{2}\right), P_{3}\left(X_{3}, Y_{3}, Z_{3}\right)$ and assuming $Z_{1}=Z_{2}=Z_{3}=0$, we obtain:

$$
\begin{aligned}
& X=\frac{X_{1}^{2}-X_{2}^{2}+L_{2}^{2}-L_{1}^{2}}{2 \cdot\left(X_{1}-X_{2}\right)}, \\
& Y=\frac{Y_{1}+Y_{3}}{2} \cdot \frac{X_{1}^{2}-X_{3}^{2}+L_{3}^{2}-L_{1}^{2}}{2 \cdot\left(Y_{1}-Y_{2}\right)} \cdot \frac{\left(L_{2}^{2}-L_{1}^{2}+X_{1}^{2}-X_{2}^{2}\right) \cdot\left(X_{3}-X_{1}\right)}{2 \cdot\left(X_{1}-X_{2}\right) \cdot\left(Y_{1}-Y_{3}\right)}
\end{aligned}
$$

and

$$
Z=\sqrt{L_{1}^{2}-\left(X-X_{1}\right)^{2}-\left(Y-Y_{1}\right)^{2}} .
$$

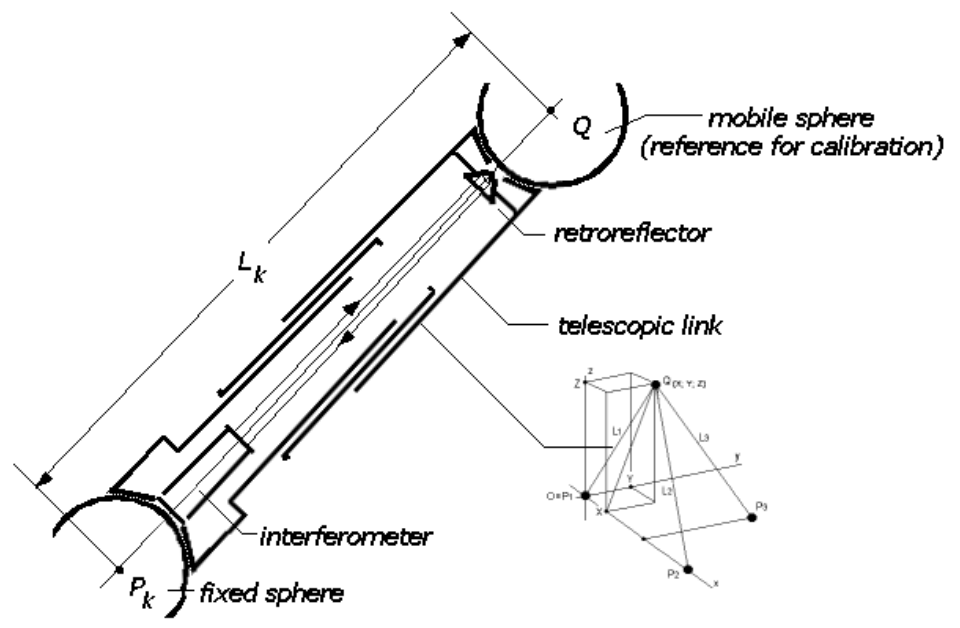

Figure 8. Telescopic links.

Or, particularly, if we consider the specific coordinates from figure 7 , $P_{1}(0,0,0), P_{2}\left(X_{2}, 0,0\right)$ and $P_{3}\left(X_{3}, Y_{3}, 0\right)$, we get:

$$
\begin{aligned}
& X=\frac{L_{1}^{2}-L_{2}{ }^{2}+X_{2}^{2}}{2 \cdot X_{2}}, \\
& Y=\frac{X_{3}^{2}+Y_{3}^{2}+L_{1}^{2}-L_{3}^{2}-X_{3} \cdot \frac{L_{1}^{2}-L_{2}^{2}+X_{2}^{2}}{X_{2}}}{2 \cdot Y_{3}}
\end{aligned}
$$

and

$$
Z=\sqrt{L_{1}^{2}-X^{2}-Y^{2}} .
$$


We use those equations in order to find reference values for the artefact and the first set of equations to find, following the GUM, the uncertainty for the reference coordinates $X, Y$ and $Z$ knowing the uncertainties for the center of the fixed spheres $X_{1}, Y_{1}, Z_{1}, X_{2}, Y_{2}, Z_{2}, X_{3}, Y_{3}$ and $Z_{3}$, and also for the length of the links $L_{1}, L_{2}$ and $L_{3}$.

Following the GUM, the uncertainty $u_{Y}$ for a dependent measure $Y=Y\left(X_{1}, X_{2}, X_{3}, \ldots, X_{n}\right)$, a function of $n$ uncorrelated measurements $X_{i}$ with uncertainties given by $u_{X i}$, is defined by:

$$
u_{Y}=\sqrt{\sum_{i=1}^{n}\left(\frac{\partial Y}{\partial X_{i}} \cdot u_{X i}\right)^{2}} .
$$

In order to bypass the derivatives $\frac{\partial Y}{\partial X_{i}}$, we are using the difference resulting from increments on $X_{i}$ equal to the uncertainty $u_{X i}$. Consequently, $u_{Y}$ is given by:

$$
u_{Y}=\sqrt{\sum_{k=1}^{n}\left[Y\left(X_{1}, X_{2}, \ldots, X_{k}, \ldots X_{n}\right)-Y\left(X_{1}, X_{2}, \ldots, X_{k}+u_{X k}, \ldots X_{n}\right)\right]^{2}} .
$$

Two problems exist relating to the length of each link $L_{i}$ and also to the coordinates for the fixed spheres $P_{1}\left(X_{1}, Y_{1}, Z_{1}\right), P_{2}\left(X_{2}, Y_{2}, Z_{2}\right)$ and $P_{3}\left(X_{3}, Y_{3}, Z_{3}\right)$.

With the laser interferometers we only are measuring length variation. We need to estimate the real length for the links, and also the coordinates for fixed spheres. We assume that $X_{1}=Y_{1}=Z_{1}=Y_{2}=Z_{2}=Z_{3}=0$, using the reference coordinates from figure 7 . All the other values, and also the uncertainties for all values, are calculated by self-calibration.

\section{Self-calibration of the Artefact}

Self-calibration (system identification) is done by moving the mobile sphere over the plane of the fixed spheres and minimizing the cost function $c$ :

$$
c=\sum_{k=0}^{m}\left(a_{1_{-} k}+a_{2_{-} k}+a_{3_{-} k}\right)
$$

where $a_{1 \_k}, a_{2 \_}, a_{3 \_k}$ are given by the set of $3(m+1)$ equations, for $k=0, \ldots, m$ :

$a_{1 \_k}=\left(X_{k_{-} e s t}-X_{1_{-} e s t}\right)^{2}+\left(Y_{k_{-} e s t}-Y_{1_{-} e s t}\right)^{2}+\left(Z_{k_{-} e s t}-Z_{1_{-} e s t}\right)^{2}-\left(L_{1_{-} e s t}+d_{1_{-} k}\right)^{2}$

$a_{2 \_k}=\left(X_{k_{-} e s t}-X_{2_{-} e s t}\right)^{2}+\left(Y_{k_{-} e s t}-Y_{2 \_e s t}\right)^{2}+\left(Z_{k_{-} e s t}-Z_{2_{-} e s t}\right)^{2}-\left(L_{2 \_e s t}+d_{2 \_k}\right)^{2}$

$a_{3_{-} k}=\left(X_{k_{-} e s t}-X_{3_{-} e s t}\right)^{2}+\left(Y_{k_{-} e s t}-Y_{3_{-} e s t}\right)^{2}+\left(Z_{k_{-} e s t}-Z_{3_{-} e s t}\right)^{2}-\left(L_{3_{-} e s t}+d_{3_{-} k}\right)^{2}$

with $X_{k \_s t}, Y_{k \_s t}, Z_{k \_s t}$ the estimated coordinates of the mobile sphere in the $k$ th position, $L_{1 \_ \text {est }}, L_{2 \_ \text {est }}, L_{3 \_ \text {est }}$ the estimated lengths for the three links at the initial 
position for $k=0$, and $d_{1 \_k}, d_{2 \_k}, d_{3 \_k}$ the measured length variations of the three links.

For $m+1$ positions of the mobile sphere, we get $3(m+1)$ equations with equal number of measurements and with $3 m+15$ unknowns $(3 m+3$ coordinates for the mobile sphere positions, 9 coordinates for the fixed spheres position and 3 initial lengths for the links).

The problem has many solutions, and the fixed sphere's location enables a quick evaluation of the validity of the solution. In figure 9 we present four possible solutions for the same inputs, resulting from the evident axis symmetrical solutions. The optimal solution search is done using the optimization toolbox of Matlab: starting with a constrained method in order to fix the solution in the adequate region and concluding with an unconstrained method to obtain a quicker and better solution.
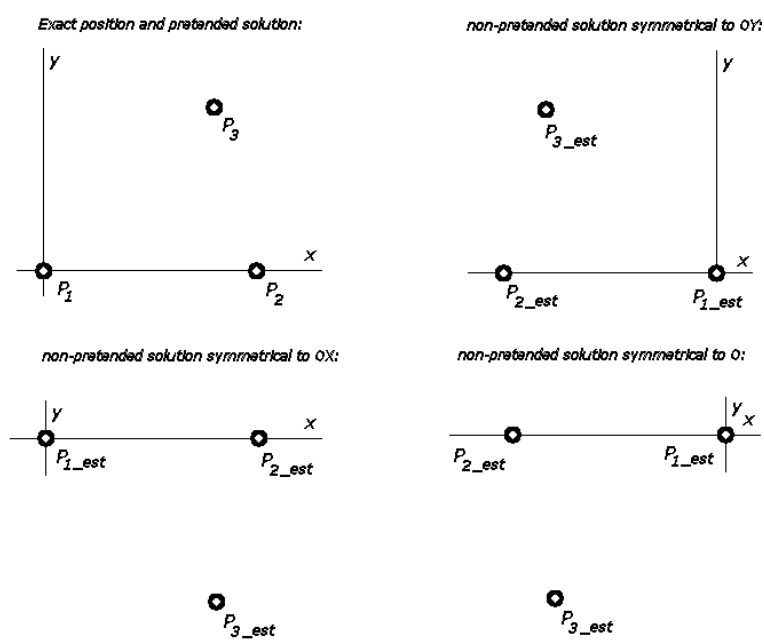

Figure 9. Those figures present possible evident solutions for self-calibration.

The cost function value is used to obtain the estimated uncertainty for the estimated parameters.

\section{Simulating Calibration Artefact}

In order to verify the adequacy of use of the developed device, some numerical simulations were made, using the software Matlab (version 6.5 R13). The programs are divided into two major parts: in the first one, the purpose is to 
realize the self-calibration of the system and, in the second, using the results for the adjusted parameters obtained in the first part, using trilateration, estimated coordinates and the correspondent uncertainty are obtained for some reference points.

The program needs initial parameters that are the minimum and the maximum link length, the link's length variation noise amplitude, the number of constrained steps (necessary to constrain the area where the solutions could be found, using the optimization method) and forbidden area radius (where no points can be placed, corresponding to a physical limitation of the artefact). Table 1 summarizes the parameters (in relative units) introduced in the presented examples.

\begin{tabular}{|c|c|c|c|c|}
\hline & & \multicolumn{3}{|c|}{ Simulation } \\
\hline & & $1^{\mathrm{st}}$ & $2^{\text {nd }}$ & $3^{\text {rd }}$ \\
\hline \multirow{4}{*}{ 营 } & maximum link length & 20 & 20 & 20 \\
\hline & links length noise amplitude & 0 & 0.01 & 0.30 \\
\hline & constrained steps & 5 & 5 & 5 \\
\hline & forbidden area radius & 0.6 & 0.6 & 0.6 \\
\hline
\end{tabular}

After the introduction of the initial parameters, the user chooses, in a graphical way using the mouse pointer, the three fixed spheres' positions and the various moving sphere positions. In figure 10 these positions are presented for the $2^{\text {nd }}$ simulation, similar to the other two simulations realized (note that in the space of measurement there are some regions of shadow, corresponding to zones that are unreachable by the moving sphere). As the user introduces a new position, the parameters are recalculated using all information available.

After introducing all moving sphere positions, the first part of the program is finished and the results of self-calibration are presented, in a graphical and in a numerical form. In table 2 we present the final numerical results of self-calibration for the $2^{\text {nd }}$ simulation corresponding to links length noise amplitude equal to 0.01 .

The results, in a graphical form, are presented in figure 11, which shows the real positions versus estimated positions of the fixed points, the variations of the link lengths, the evolution of the cost function that is minimized during the program and the evolution of the estimated values for the initial link lengths.

For the $1^{\text {st }}$ and the $3^{\text {rd }}$ simulations the graphical results of self-calibration are shown in figures 12 and 13. 


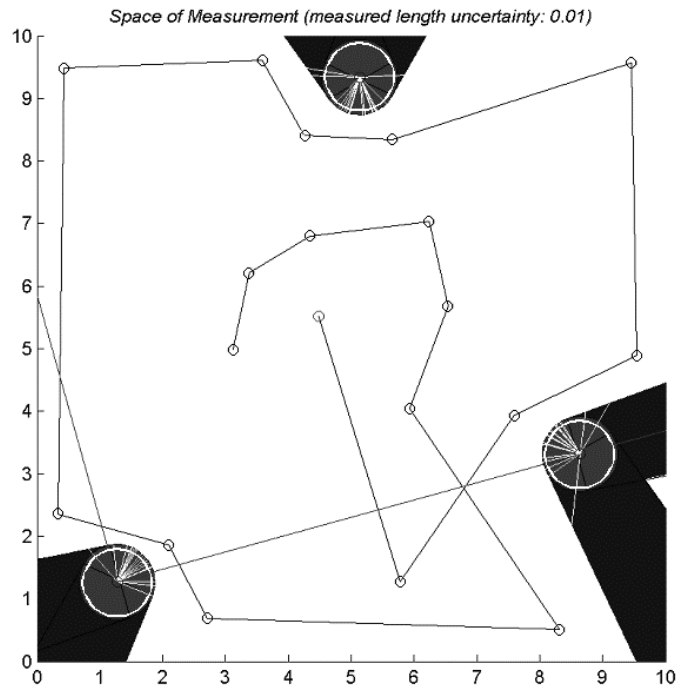

Figure 10. Example of fixed spheres position and mobile sphere trajectory during self-calibration.

Table 2. Results of self-calibration with links length noise amplitude 0.01 .

\begin{tabular}{|c|c|c|c|}
\cline { 2 - 4 } \multicolumn{1}{c|}{} & reference value & estimated value & deviation \\
\hline & 4.14524605719157 & 4.14762057356121 & 0.00237451636964 \\
initial links lengths & 5.77216978117969 & 5.76981912447491 & -0.00235065670478 \\
& 4.80120164387303 & 4.81250457805668 & 0.01130293418365 \\
\hline$x$ of $2^{\text {nd }}$ fixed point & 7.64741472782958 & 7.64545798330029 & -0.00195674452929 \\
$x$ of $3^{\text {rd }}$ fixed point & 5.87877399052966 & 5.89051558442518 & 0.01174159389553 \\
$y$ of $3^{\text {rd }}$ fixed point & 6.74274716485670 & 6.75540982406257 & 0.01266265920588 \\
\hline $1^{\text {st }}$ moving point $x$ & 2.76878359671112 & 2.77157430333704 & 0.00279070662592 \\
$1^{\text {st }}$ moving point $y$ & 3.08494769311341 & 3.08807624576027 & 0.00312855264686 \\
\hline $2^{\text {nd }}$ moving point $x$ & 3.35102638810543 & 3.36071345716409 & 0.00968706905866 \\
$2^{\text {nd }}$ moving point $y$ & 4.19778270569769 & 4.19928162592284 & 0.00149892022515 \\
\hline$\ldots$ & $\ldots$ & $\ldots$ & $\ldots$ \\
\hline
\end{tabular}

After the self-calibration, it is possible to introduce in the program some values of $3 \mathrm{D}$ reference points coordinates and compare them with the correspondent estimated coordinates, obtained from the final parameters (given by the self-calibration process) using trilateration. In addition, those results have a corresponding estimated uncertainty, a function of the uncertainty of the links' 
lengths in table 3 are summarized some results for 4 reference points obtained in the three simulations realized.
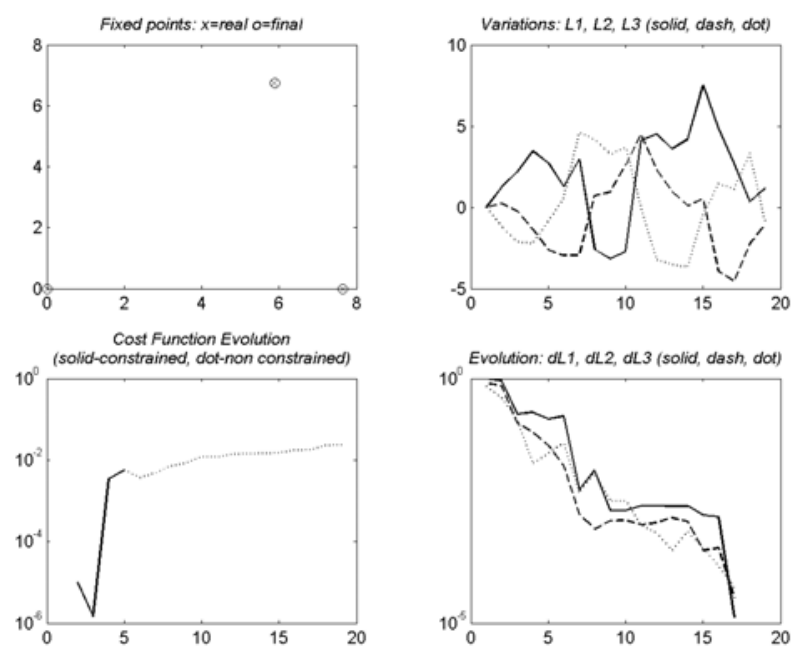

Figure 11. Self-calibration with link's length noise amplitude $=0.01$.
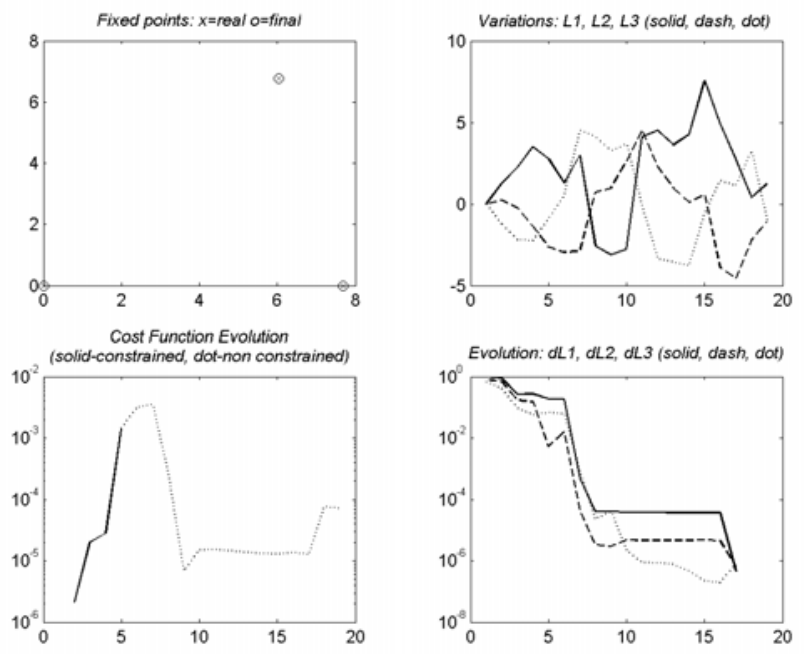

Figure 12. Self-calibration with link's length noise amplitude $=0.0$. 

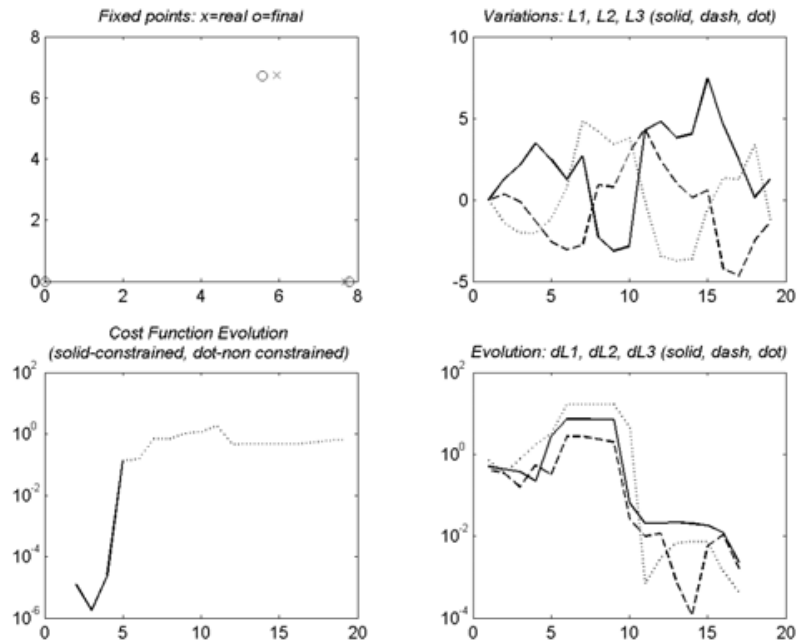

Figure 13. Self-calibration with link's length noise amplitude $=0.3$.

Table 3. Trilateration results.

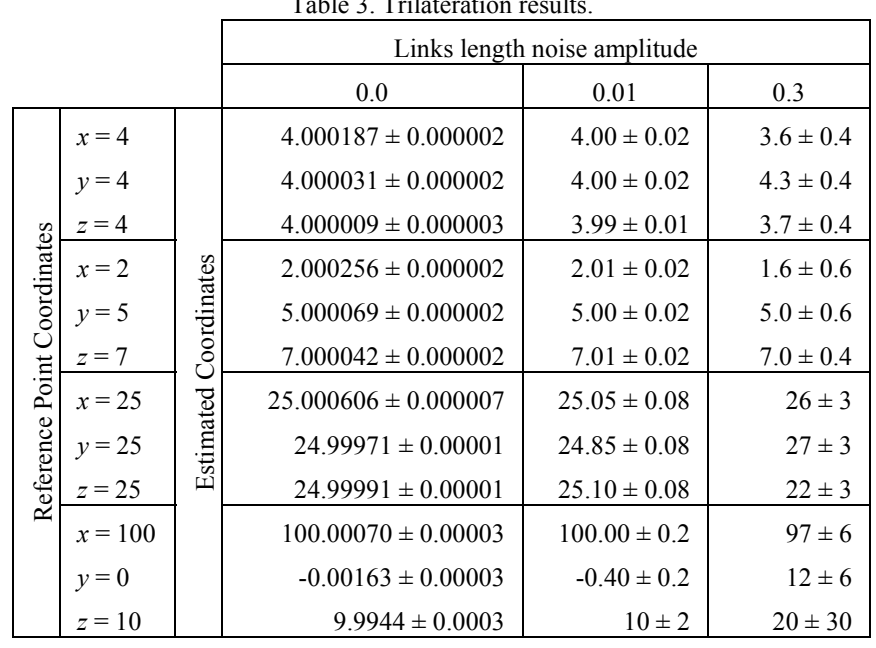




\section{Reference Values for CMM Calibration - Uncertainty Propagation}

Coordinates, and uncertainties for those coordinates, are the reference values for CMMs calibration. The coordinates and the respective uncertainties are calculated in real time, by a method based on laser interferometer measurement of the links length variation.

For that purpose, we tried several Data Fusion solutions such as the Kalman Filter, the Extended Kalman Filter, the covariance intersection, etc. In this paper, we present the results obtained using the standard definition for uncertainty propagation (due to its extension and unsolved small issues, further results will be presented later).

In figure 14, we represent some of the estimated uncertainty values for $X, Y$ and $Z$ coordinates of the moving sphere in some regions of the measurement space, for a link length noise amplitude equal to 0.01 . As we can see, the final uncertainty in each coordinate, not only depends on the link length uncertainty value, but also depends on the value of that coordinate and on the values of the other two coordinates.

From figure 14, we can also see that the uncertainties of $X$ and $Y$ slowly increase when the coordinate $Z$ from the mobile sphere increases. The uncertainty for the $Z$ coordinates increases during trilateration for small values of $Z$ and reduces when $Z$ is increasing. Those values are not acceptable and trilateration only may be used for values of $Z$ not equal to 0 . When the mobile sphere is over the plane of the fixed spheres, only the $X$ and $Y$ coordinates must be calibrated, as in the case of using the ball plate artefact.
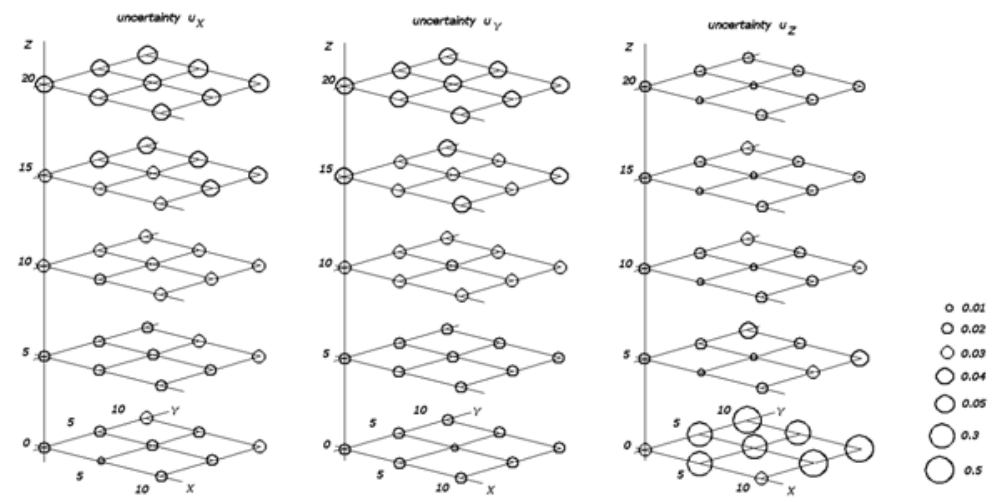

Figure 14. Moving sphere coordinates uncertainty in several regions of the measurement space. 


\section{Conclusions}

The measurements made with the use of the described artefact have errors and a previously unknown uncertainty. In this text, above, we presented a technique to incorporate the measured values uncertainties in order to obtain the calibration results' final uncertainties (using data fusion as a learning system, meaning that the optimal uncertainty estimation is constantly adapted during self-calibration of the device). However, this is not the final answer for this problem of artefact validation: we are developing and comparing different techniques in non-linear and non-observable state estimation.

The results obtained for the final calibration uncertainty using those techniques lead us to the following conclusions. First, in order to decide among the various techniques, we need to take into account the amount of computer memory required for identification, the accuracy of the method, the difficulties in implementation and also the ability to represent multimodal probability density functions (PDFs).

In table 4, where $K F$ represents the Kalman filter, $E K F$ represents the extended Kalman filter, $C I$ represents the covariance intersection, $C P$ represents the covariance propagation, Markov represents the interval functions with fixed cells, probability grids or maximum entropy estimation and, finally, $P F$ represents various particle filters (the set constituted by Monte Carlo localization, bootstrap, condensation algorithm and fittest algorithm), we summarize the comparison of the different techniques studied.

\begin{tabular}{|c|c|c|c|c|c|c|} 
Table 4. Comparing calculating data fusion techniques for uncertainty. \\
\cline { 2 - 7 } & $K F$ & $E K F$ & $C I$ & $C P$ & Markov & $P F$ \\
\hline $\begin{array}{c}\text { Starting geometry } \\
\text { knowledge }\end{array}$ & \multicolumn{2}{|c|}{ good estimation } & \multicolumn{4}{|c|}{ from scratch } \\
\hline Time of response & more & more & more & more & more & less \\
\hline $\begin{array}{c}\text { Memory } \\
\text { consuming }\end{array}$ & less & less & less & less & more & less \\
\hline $\begin{array}{c}\text { Ability to represent } \\
\text { multimodal PDFs }\end{array}$ & less & less & more & more & more & more \\
\hline
\end{tabular}

As a conclusion, we can state that the particle filters seem to be the best methods for our goal. However, the ability to represent multimodal PDFs seems to be very restrictive and a better analysis need to be realized taking into 
account the costs involved (time response, memory consuming, etc.). In other way, the Kalman filter and the extended Kalman filter need a starting geometry knowledge that can be estimated, measuring the position of fixed points and changing coordinates following the artefact coordinate system.

The results given by using the developed Matlab routines based on the above make us conclude that the self-calibration of the artefact works well. The modelling of the artefact allows us to develop the real artefact that we are starting to build and, in the near future, we will have results from that artefact.

\section{References}

1. S. D. Antunes, P. M. Antunes and M. A. F. Vicente; Local and Global Calibration of Coordinate Measuring Machines; in Advanced Mathematical and Computational Tools in Metrology V; Series on Advances in Mathematics for Applied Sciences Vol.57, World Scientific (2001) pp 16-22.

2. L. Arriba, E. Trapet, M. Bartscher, M. Franke, A. Balsamo, G. Costelli, S. Torre, F. Kitzsteiner and F. San Martín; Methods and Artifacts to Calibrate Large CMMs, in Proceedings of the $1^{\text {st }}$ international EUSPEN conference, Bremen, Germany (1999) pp 391-394.

3. M. Bartscher, K. Busch, H. Schwenke, E. Trapet and F. Wäldele; Artefact based Traceability Concept for Large Co-ordinate Measuring Machines $(C M M S)$, in Proceedings of the $9^{\text {th }}$ METROLOGIE Conference, Bordeaux, France (1999) pp 158-161

4. .ISO; Guide to the Expression of Uncertainty in Measurements (GUM), Geneva, Switzerland (1995).

5. J. F. Silva; Artefactos para a calibração de máquinas de medir por coordenadas, rapport of Projecto de Produção, under the supervision of S. D. Antunes, IST-DEM-STM (2001). 